\title{
Atypical Fabry's Disease Presenting with Cholesterol Crystal Embolization
}

\author{
Toshihiro Shirai, Takayasu Ohtake, Masato Kimura*, Masatoshi Iwata**, Yoshihide FujIgaki***, \\ Shigekazu TaKayanagi, Kingo ChIDA ${ }^{* * * *}$, Hirotoshi NAKamura $* * * *$, Akira HishidA $* * *$ and Fumitoshi IRIE*****
}

\begin{abstract}
We describe a 65-year-old man who presented with pulmonary hemorrhage and progressive renal insufficiency three months after resection surgery for an abdominal aortic aneurysm. Intensive treatment with corticosteroids and hemodialysis were not effective, and the patient died. Postmortem examination of the kidneys revealed widespread cholesterol clefts within the renal arterioles and a number of lamellar inclusion bodies were observed by electron microscopy. The diagnosis of Fabry's disease was made by the absence of plasma $\alpha$-galactosidase $A$ activity. This was a very rare case of subclinical Fabry's disease coexistent with cholesterol crystal embolization, mimicking pulmonary-renal syndrome.
\end{abstract}

(Internal Medicine 39: 646-649, 2000)

Key words: $\alpha$-galactosidase A, glycosphingolipid, pulmonary hemorrhage, pulmonary-renal syndrome

\section{Introduction}

Fabry's disease is an X-linked recessive disease resulting from a deficiency of a lysosomal enzyme, $\alpha$-galactosidase A, leading to the progressive accumulation of glycosphingolipids, predominantly globotriaosylceramide in the vascular endothelial and smooth muscle cells throughout the body (1). In hemizygous male patients with nondetectable $\alpha$-galactosidase A activity, angiokeratomas, acroparesthesias, hypohidrosis, and corneal opacities are observed in childhood or adolescence; later in adulthood, renal, cerebral, or myocardial vascular disease may also occur. Unless hemodialysis or renal transplantation is undertaken, death usually occurs from uremia in the fourth decade. Heterozygous females are usually asymptom- atic and have a normal life span. In contrast, atypical hemizygotes have been described with residual $\alpha$-galactosidase A activity and milder disease manifestations, mostly limited to the myocardium or kidney (2-7).

For editorial comment, see p 601.

Cholesterol crystal embolization usually occurs in elderly males as a complication of atherosclerosis (8). Predisposing factors include catheterization procedures, vascular surgery, and the use of anticoagulants. Although the kidney and the skin are the two most commonly involved organs, the clinical manifestations vary, mimicking multisystem disorders such as systemic vasculitis. The premortem diagnosis of the disorder, which relies primarily on clinical suspicion, is often difficult to make. The mortality rate is as high as $80 \%$ because effective therapy has not been established.

The coexistence of Fabry's disease with cholesterol crystal embolization has not been reported in the literature. We herein describe a 65-year-old man who presented with progressive renal failure and pulmonary hemorrhage. Intensive treatment with corticosteroids for pulmonary-renal syndrome was not effective. The pathological findings in kidney specimens at autopsy were consistent with Fabry's disease and cholesterol crystal embolization. This was an unusual case of a Fabry hemizygote who was essentially asymptomatic until the age of 65 when cholesterol crystal embolization occurred. The clinical features of the pulmonary hemorrhage were also unusual.

\section{Case Report}

A 65-year-old man was admitted to our hospital with exertional dyspnea, which had increased progressively for five days, and mild hemoptysis. The patient had had a cerebral infarction 10 years before admission and had a 10-year history of hypertension and diabetes mellitus, but no hyperlipidemia.

From the Department of Internal Medicine, Fujinomiya City General Hospital, Fujinomiya, *University of Shizuoka School of Nursing, Shizuoka, **the Department of Internal Medicine, Haibara General Hospital, Haibara, ***the First and ****the Second Departments of Internal Medicine, Hamamatsu University School of Medicine, Hamamatsu and *****the Laboratory for Cellular GlycoBiology, The Institute of Physical and Chemical Research (RIKEN)

Received for publication August 19, 1999; Accepted for publication January 4, 2000

Reprint requests should be addressed to Dr. Toshihiro Shirai, the Department of Internal Medicine, Fujinomiya City General Hospital, 3-1, Nishiki-cho, Fujinomiya 418-0076 
Aspirin was administered to prevent the recurrence of cerebral infarction, hypertension was controlled with nifedipine, and diabetes mellitus was treated by diet. Until 10 years before he had had no serious illness and there was no history of acroparesthesias or hypohidrosis since childhood. His father died of a rupture of an abdominal aortic aneurysm, while his mother, six siblings, one son and two daughters were in good health. He also had an abdominal aortic aneurysm, which had been found by chance, and three months before admission, he underwent resection and graft replacement surgery. His baseline creatinine value was $0.63 \mathrm{mg} / \mathrm{dl}$ and urinalysis showed only glucosuria, but no proteinuria. The creatinine value had risen to $1.47 \mathrm{mg} / \mathrm{dl}$ two months after the vascular surgery. Physical examination on admission revealed diminished vesicular sound in the right lung and right hemiparesis. Blood pressure was $117 / 84 \mathrm{mmHg}$. Temperature was $37.5^{\circ} \mathrm{C}$. No corneal opacity or angiokeratoma was observed on close examination. Laboratory data revealed the following: blood urea nitrogen, 34.9 $\mathrm{mg} / \mathrm{dl}$; serum creatinine, $3.1 \mathrm{mg} / \mathrm{dl}$; hemoglobin, $9.8 \mathrm{~g} / \mathrm{dl}$; hematocrit, $29.7 \%$; WBC, $8,600 / \mu 1$ with $67 \%$ neutrophils and $7 \%$ eosinophils; thrombocytes, $223,000 / \mu \mathrm{l}$; prothrombin time, 13.2 s (\% activity, 90\%); activated partial thromboplastin time, 39.4 $\mathrm{s}$; and erythrocyte sedimentation rate, $82 \mathrm{~mm}$ at 1 hour. Urinalysis showed $2+$ protein and the sediment contained rare RBCs, WBCs, and casts. Arterial blood gas values on room air showed a pH of 7.42; $\mathrm{PaCO}_{2}, 34$ Torr; $\mathrm{PaO}_{2}, 58$ Torr; and an oxygen saturation value of $89 \%$. A chest radiography showed alveolar infiltrates in the right lung field. An ECG demonstrated sinus tachycardia but otherwise showed no abnormalities.

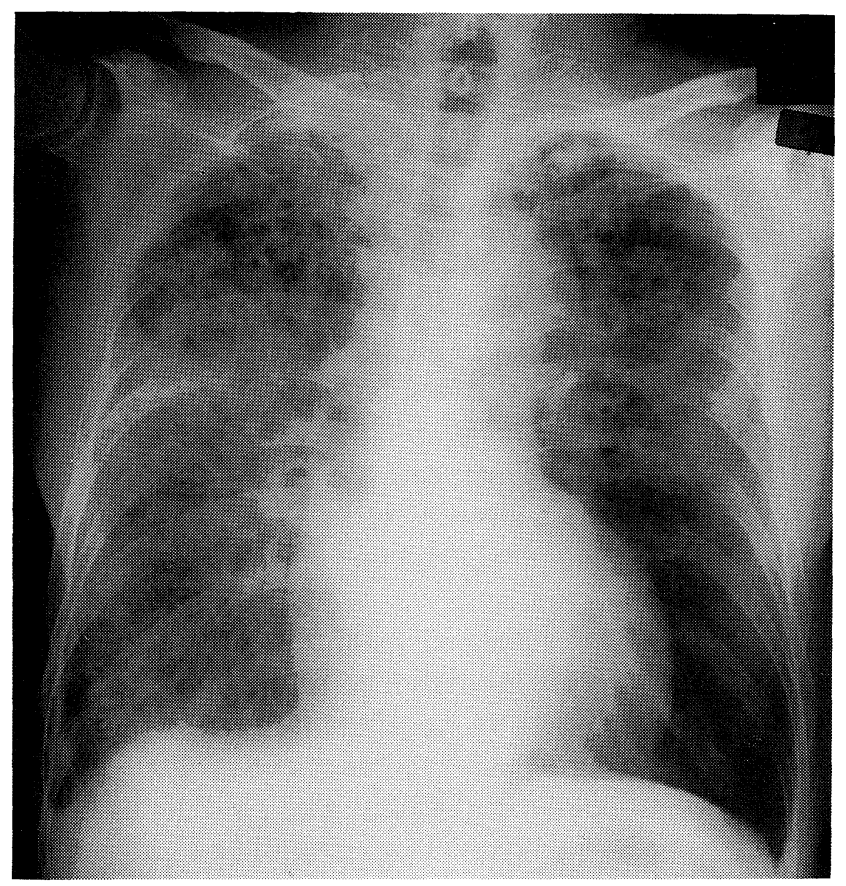

Figure 1. Chest radiography on the 3rd hospital day showing bilateral infiltrates.
Treatment with broad-spectrum antibiotics was initiated for presumed pneumonia. Three days after admission, he developed respiratory distress, requiring intubation and ventilatory support. Bronchoscopy revealed blood throughout the airways; however, endobronchial lesions were not detected. Chest radiography revealed evolving bilateral infiltrates (Fig. 1). Cultures of bronchial washings, blood and urine were negative. Corticosteroid therapy with methylprednisolone, $1 \mathrm{~g} / \mathrm{day}$, and subsequent prednisolone, $60 \mathrm{mg} /$ day, was started for presumed pulmonary-renal syndrome. On the 6th hospital day, he became comatose. A brain computed tomography scan showed only the old infarction without hemorrhage and a physical examination revealed purple discoloration of several toes. The blood urea nitrogen and creatinine levels were increased to $140.8 \mathrm{mg} / \mathrm{dl}$ and $9.1 \mathrm{mg} / \mathrm{dl}$, respectively. Hemodialysis treatment was started on the 7th day. Immunoglobulin $\mathrm{G}$ was 2,114 $\mathrm{mg} / \mathrm{dl}$, IgA was $327 \mathrm{mg} / \mathrm{dl}$, and IgM was $61 \mathrm{mg} / \mathrm{dl}$. The complement levels were within the normal range. Serum antinuclear antibody, cryoglobulins, myeloperoxidase-antineutrophil cytoplasmic antibody (ANCA), proteinase 3-ANCA, antiglomerular basement membrane antibody, and circulating immune complexes were all negative. His respiratory status improved slightly and renal function was stabilized after 5 hemodialysis sessions; however, he was comatose and cardiac function was worsening. On the 14th hospital day, the patient had a cardiac arrest and died.

At autopsy, there were severe atherosclerotic changes in the aorta and the coronary arteries. Examination of the kidneys revealed widespread cholesterol clefts within the renal arterioles consistent with cholesterol crystal embolization (Fig. 2). Furthermore, vacuolization was present in the cells of the kidneys, particularly the glomerular epithelial cells. Corresponding to the vacuolization, a number of lamellar inclusion bodies were observed by electron microscopy, which strongly suggested Fabry's disease (Fig. 3). Examination of the lungs

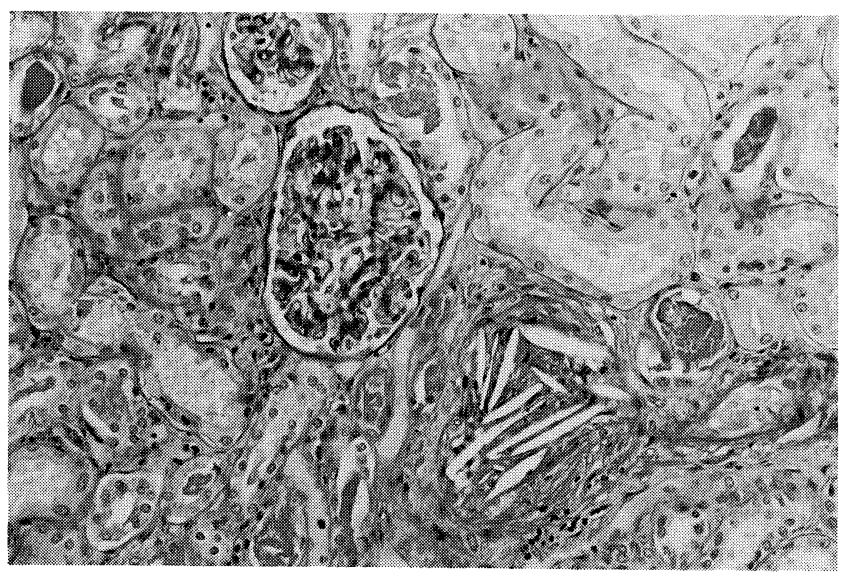

Figure 2. Histological appearance of the kidney showing an interlobular artery occluded by cholesterol crystals (HE stain, $\times 150)$. 


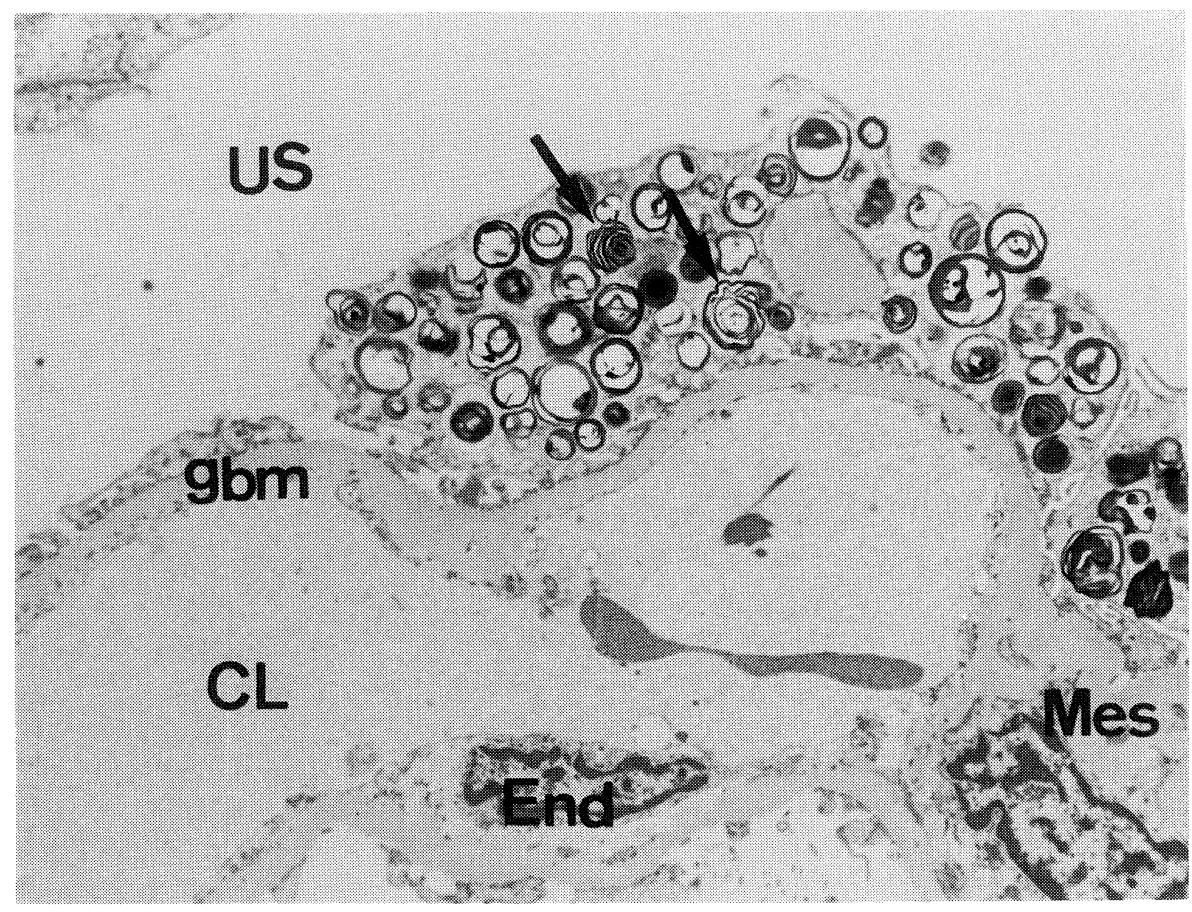

Figure 3. Electron micrograph showing osmiophilic laminated structures (arrows) in a swollen glomerular epithelial cell $(\times 4,000)$. US: urinary space, gbm: glomerular basement membrane, CL: capillary lumen, End: endothelial cell, Mes: mesangial cell.

showed some airspace exudate and hemorrhage as well as zones of capillary proliferation in some of the cells; however, cholesterol clefts were not found in the pulmonary or bronchial arteries. Electron microscopy of the lungs showed no lamellar inclusions in the pulmonary vasculature. There was no cardiomegaly or ventricular hypertrophy in the heart, although lamellar bodies were found in the myocardial cells. Examination of the brain was not performed because of the family's refusal.

To confirm the diagnosis of Fabry's disease, $\alpha$-galactosidase A activity in the serum was measured by the method using p-nitrophenyl- $\alpha$-galactoside as a substrate. No activity was detected in the serum of the patient. In contrast, $\beta$-galactosidase activity, which was assayed simultaneously as a control, was within the normal range. Unfortunately, assays of $\alpha$-galactosidase A activity in the patient's leukocytes and examinations of the family members could not be done.

\section{Discussion}

So-called pulmonary-renal syndrome refers to a variety of disorders that are characterized by pulmonary hemorrhage and progressive renal insufficiency $(9-11)$. These include Goodpasture's syndrome, Wegener's granulomatosis, microscopic polyarteritis, systemic lupus erythematosus, mixed cryoglobulinemia, and Henoch-Schölein purpura. Although systemic vasculitides are the main causes of pulmonary-renal syndrome, there are conditions that can mimic vasculitis, including thromboembolic diseases, infections, neoplastic diseases such as atrial myxoma, cholesterol crystal embolization (atheromatous emboli), and miscellaneous diseases such as Fabry's disease (12). Rare cases of cholesterol crystal embolization or Fabry's disease that presented with pulmonary and renal involvement have been reported (13-16). However, the present case was extremely rare in that both diseases coexisted.

Classic Fabry hemizygotes have no detectable $\alpha$-galactosidase A activity, while atypical variants have residual activity compatible with their milder manifestations. These atypical variants have residual activity in plasma, urine, leukocytes, fibroblasts, or other tissues. Occasionally, rare cases have been reported in which activity in plasma was markedly decreased, while the activity in leukocytes, or fibroblasts was within the normal range $(3,6)$. This phenomenon is thought to be due to stability mutation of the enzyme. The present patient had no typical symptoms or signs of Fabry's disease until the age of 65 except for the cerebral infarction which occurred at the age of 55. Although $\alpha$-galactosidase A activity in the serum was nondetectable and residual activity in cells or tissues could not be demonstrated, we believe that our case was an atypical variant of Fabry's disease. With respect to the past history of cerebral infarction, classic hemizygotes or even atypical variants have not been described in patients who presented with only cerebral infarctions without other manifestations. Atherosclerosis might be the etiological factor for the development of the cerebral infarction in our case.

Most atypical hemizygotes of Fabry's disease have cardiac manifestations including cardiomegaly and cardiomyopathy, 
with mild or no proteinuria. Recently, atypical cases with only proteinuria or subclinically coexisting with IgA nephropathy have been reported (5-7). In these renal variants, characteristic ultrastructural findings could be detected in the kidneys or urinary sediment by electron microscopy, even when their renal function was within the normal range. In the present case, renal autopsy revealed findings consistent with cholesterol embolization as well as Fabry's disease. His creatinine value was within the normal range and urinalysis showed no proteinuria before the vascular surgery, which might have promoted the onset of cholesterol embolization. In fact, the renal function worsened gradually after the surgery, leading to renal insufficiency after three months. Therefore, we consider our case as a subclinical case of atypical Fabry hemizygote, and cholesterol embolization as the main cause of the progressive renal insufficiency.

Retrospectively, a prior history of atherosclerotic diseases, the predisposing factor of vascular surgery procedures, and clinical manifestations of progressive renal insufficiency and dermatological lesion of "purple toes" were consistent with cholesterol crystal embolization and the diagnosis was confirmed by renal autopsy findings. The factor that made the premortem diagnosis difficult was the presentation of pulmonary hemorrhage. In general, cholesterol crystal embolization is rarely accompanied by pulmonary hemorrhage in the absence of an arteriovenous fistula (13). In pathologically proven cases, cholesterol crystals were found in the pulmonary and bronchial arteries of biopsied lung specimens. It has been suggested, however, that glycosphingolipid accumulation in the pulmonary vascular smooth muscle or endothelial cells may cause hemoptysis, and that there is pathological evidence of vascular involvement in some patients with Fabry's disease $(17,18)$. However, our postmortem examination failed to show the cholesterol clefts or Fabry's ultrastructural findings by electron microscopy in the pulmonary vasculature. Whether the capillary proliferation found in our case was due to Fabry's disease or to secondary changes of cholesterol embolization or to other causes could not be determined. The cause of pulmonary hemorrhage is unknown.

No examination of the brain was performed, so we could not assess the cause of coma. The simultaneous occurrence of coma and the skin lesion of "purple toes", however, suggests that cholesterol crystal embolization but not Fabry's disease, was associated with the comatose state in our patient. Similarly, although lamellar bodies were found in the myocardial cells, it is unlikely that Fabry's disease was the direct cause of death because of the absence of ventricular hypertrophy or a history of cardiac problems.

Although the association of Fabry's disease and cholesterol crystal embolization may be incidental but not causal, the clinical features were identical to those of pulmonary-renal syndrome. Fabry's disease and cholesterol crystal embolization should be considered in the differential diagnosis of pulmonary-renal syndrome.

\section{References}

1) Desnick RJ, Ioannou YA, Eng CM. $\alpha$-galactosidase A deficiency: Fabry disease. in: The Metabolic and Molecular Bases of Inherited Disease. 7th ed. Scriver CR, Beaudet AL, Sly WS, Valle D, Eds. McGraw-Hill, New York, 1995: 2741-2784.

2) Romeo G, D'Urso M, Pisacane A, Blum E, De Falco A, Ruffilli A. Residual activity of $\alpha$-galactosidase A in Fabry's disease. Biochem Genet 13: 615-628, 1975.

3) Bishop DF, Grabowski GA, Desnick RJ. Fabry disease: an asymptomatic hemizygote with significant residual $\alpha$-galactosidase $A$ activity. Am J Hum Genet 33: 71A, 1981 (Abstract).

4) von Scheit W, Eng CM, Fitzmaurice TF, et al. An atypical variant of Fabry's disease with manifestations confined to the myocardium. N Engl J Med 324: 395-399, 1991.

5) Sawada K, Mizoguchi K, Hishida A, et al. Point mutation in the $\alpha$-galactosidase A gene of atypical Fabry disease with only nephropathy. Clin Nephrol 45: 289-294, 1996.

6) Kawamura O, Sakuraba H, Itoh $\mathrm{K}$, et al. Subclinical Fabry's disease occurring in the context of IgA nephropathy. Clin Nephrol 47: 71-75, 1997.

7) Meroni $M$, Spisni $C$, Tazzari $S$, et al. Isolated glomerular proteinuria as the only clinical manifestation of Fabry's disease in an adult male. Nephrol Dial Transplant 12: 221-223, 1997.

8) Fine MJ, Kpoor W, Falanga V. Cholesterol crystal embolization: a review of 221 cases in the English literature. Angiology 38: 769-784, 1987.

9) Schwarz MI, Cherniack RM, King TE. Diffuse alveolar hemorrhage and other rare infiltrative disorders. in: Textbook of Respiratory Medicine. 2nd ed. Murray JF, Nadel JA, Eds. Saunders, Philadelphia, 1994: 1889_ 1912.

10) Green RJ, Ruoss SJ, Kraft SA, Duncan SR, Berry GJ, Raffin TA. Pulmonary capillaritis and alveolar hemorrhage: update on diagnosis and management. Chest 110: 1305-1316, 1996.

11) Niles JL, Böttinger EP, Saurina GR, et al. The syndrome of lung hemorrhage and nephritis is usually an ANCA-associated condition. Arch Intern Med 156: 440-445, 1996.

12) Sack KE. When vasculitis is not vasculitis. Hosp Pract (Off Ed) 28: 94, 97-100, 103, 1993.

13) Sabatine MS, Oelberg DA, Mark EJ, Kanarek D. Pulmonary cholesterol crytal embolization. Chest 112: 1687-1692, 1997.

14) Vacher-Coponat H, Pache X, Dussol B, Berland Y. Pulmonary-renal syndrome responding to corticosteroids: consider cholesterol embolization. Nephrol Dial Transplant 12: 1977-1979, 1997.

15) Parkinson JE, Sunshine A. Angiokeratoma corporis diffusum universale (Fabry). Presenting as suspected myocardial infarction and pulmonary infarcts. Am J Med 31: 951-958, 1961.

16) Leder AA, Bosworth WC. Angiokeratoma corporis diffusum universale (Fabry's disease) with mitral stenosis. Am J Med 38: 814-819, 1965.

17) Brown LK, Miller A, Bhuptani A, et al. Pulmonary involvement in Fabry disease. Am J Respir Crit Care Med 155: 1004-1010, 1997.

18) Smith $P$, Heath $D$, Rodgers $B$, Helliwell T. Pulmonary vasculature in Fabry's disease. Histopathology 19: 567-569, 1991. 